\title{
Bildgebung in Klinik und Forschung: Beitrag zur Individualisierten Medizin?*
}

\author{
Imaging in Clinic and Research: Contribution to Individualized Medicine?
}

Autoren

Otmar Schober ${ }^{1}$, Olaf Dössel ${ }^{2}$, Helmut Ermert ${ }^{3}$, Hermann Requardt ${ }^{4}$, Sibylle Ziegler ${ }^{5}$, Gerhard Adam ${ }^{6}$

Institute

1 Medizinische Fakultät, Westfälische Wilhelms-Universität Münster

2 Institut für Biomedizinische Technik, Karlsruher Institut für Technologie

3 Fakultät für Elektrotechnik und Informationstechnik, RuhrUniversität Bochum

4 acatech - Deutsche Akademie der Technikwissenschaften, München

5 Klinik und Poliklinik für Nuklearmedizin, Klinikum der Ludwig-Maximilians-Universität München

6 Klinik und Poliklinik für Diagnostische und Interventionelle Radiologie und Nuklearmedizin, Universitätsklinikum Hamburg-Eppendorf
Korrespondenzadresse

Otmar Schober

Von-Esmarch-Straße 125, 48149 Münster

otmar.schober@uni-muenster.de

Bibliografie

DOI https://doi.org/10.1055/s-0043-115599

Online-Publikation: 2017 | Fortschr Röntgenstr 2017; 189:

939-944 @ Georg Thieme Verlag KG Stuttgart · New York ISSN 1438-9029
In den Lebenswissenschaften ist die Individualisierte Medizin aktuell ein zentrales Thema, vielleicht ein Hype. Das bestätigen auch synonyme, erweiternde und klärende Begriffe wie Personalisierte Medizin, Customized Medicine, Stratifizierende Medizin oder Präzisionsmedizin. In einer „State of the Union Address“ an die Bevölkerung der Vereinigten Staaten von Amerika hat Präsident Barack Obama 2015 die Bedeutung der Präzisionsmedizin hervorgehoben [1]. Diese Initiative wurde inhaltlich wesentlich vom Direktor des National Institute of Health, dem Genetiker Francis Collins, vorangetrieben [2]. Individualisierte Medizin, das ist eine gute Botschaft, betont sie doch die Wertigkeit des einzelnen Patienten. Für klinisch tätige Ärzte ist das bereits eine Selbstverständlichkeit. Die Bedeutung eines Wortes, und das sei mit einem Lächeln hinzugefügt, zeigt sich nach Wittgenstein im Gebrauch der Sprache [3].

Die Nationale Akademie der Wissenschaften Leopoldina, die acatech - Deutsche Akademie der Technikwissenschaften und die Union der Deutschen Akademien der Wissenschaften haben im Dezember 2014 eine Stellungnahme über Voraussetzungen und Konsequenzen der Individualisierten Medizin publiziert. Darin wird der Fokus, wie im Vorwort ausgeführt, auf molekulare, genetische und pharmakologische Aspekte der Onkologie gelegt, einen Bereich, „in dem die Individualisierung am weitesten fort-

* Dieses Editorial wird zeitgleich in Nuklearmedizin publiziert. Schober O, Dössel O, Ermert $\mathrm{H}$ et al. Bildgebung in Klinik und Forschung: Beitrag zur Individualisierten Medizin? Nuklearmedizin 2017; 56: 157 - 161; https://doi.org/10.3413/2017-05-0002. geschritten ist. Diese Beschränkung bedeutet, dass andere eng assoziierte Themen, wie die Patienten- und Versorgungsperspektiven, der Bereich der Medizintechnik oder neue Erkrankungen, beispielsweise in der Psychiatrie, in dieser Studie nicht beleuchtet werden. Die Behandlung dieser Gebiete bedarf einer separaten nachfolgenden Betrachtung." [4].

Vor einer Betrachtung wesentlicher Beiträge allein der Bildgebung in der Individualisierten Medizin seien wenige vorausschickende Bemerkungen zu zwei Grenz-Fragen der Wissenschaft gemacht. Was steht für und gegen Stellungnahmen der genannten Akademien? Erfüllt die Beschäftigung mit der Bildgebung Kriterien der Wissenschaft?

Eine der Aufgaben der Akademien ist es, Ordnung in die vorhandenen Aussagen und auch damit vorhandene Daten zu bringen, die in unterschiedlichen Disziplinen hervorgebracht werden, um daraus ein Gesamtbild zu formen. Weiterhin sollen Empfehlungen gegeben werden, wie die Entwicklungen günstig zu beeinflussen sind [5]. Wichtige Grenzen der wissenschaftlichen Politikbzw. Gesellschaftsberatung bilden etwa Katastrophenwarnungen und Heilsverheißungen, andererseits nicht-altruistische, sondern dem Eigeninteresse dienende, wie auch politisierende Verlautbarungen. Das setzt eine Selbstbegrenzung voraus.

Wünschenswert ist bei wissenschaftlichem Handeln der Respekt von und vor Grenzen, ist die Distanz zur Macht. Diese Macht kann der Politik, der Ökonomie und auch den Medien zugeschrieben werden; fatal sind Nähe oder gar Einfluss von Ideologien. Dabei wird nicht nur ein behutsamer Umgang mit Empfehlungen 
angesprochen; im wissenschaftlichen Handeln sind gerade beim Gegenstand der Bildgebung Berührungen mit der und sogar Überlappungen mit Interessen der Industrie möglich, teilweise gewünscht und fruchtbar. Letztlich ist es ja immer die Industrialisierung, die medizinischen Fortschritt, sei er pharmakologisch oder technologisch abbildbar, breiten Bevölkerungsgruppen und entlegenen Standorten zugänglich macht. Das bedarf immer einer gegenseitigen kritischen Begleitung.

Die Wissenschaft dient dem Erkenntnisgewinn. Dazu gehört eine methodische Suche nach Wahrheit, die alle Befähigten überprüfen und nachvollziehen können. Wenn dieses angenommen, akzeptiert werden kann, dann sind die Technikwissenschaften Wissenschaften, dann betreiben Radiologie und Nuklearmedizin Wissenschaft (science). Dann bedarf es nicht der Aufzählung von Nobelpreisen oder ähnlichen Anerkennungen in der Scientific Community. Dann bedarf es nicht der Aufzählung fachlicher Beispiele; die Methoden und die Technologien fallen nicht vom Himmel. Wissenschaft wird von Menschen betrieben und getragen. An Pioniere der Bildgebung, an Personen, wie Wilhelm Conrad Röntgen, Marie Curie, Godfrey Hounsfield, Peter Mansfield, Paul Lauterbur oder Stephan Hell sei erinnert.

Radiologie [6] und Nuklearmedizin [7] tragen in hohem Maße zur maßgeschneiderten Diagnostik und Therapie im Zeitalter der Präzisionsmedizin oder Individualisierten Medizin bei. Sie bilden das Fundament der klinischen Medizin. Schon jetzt ist ein Grad der Präzision erreicht, den die molekulare, genetische und pharmakologische Medizin noch erreichen soll. Die Akteure in diesem Feld werden ihre Forschungsanstrengungen mit den Partnern aus den Natur- und Ingenieurwissenschaften sowie der Industrie gemeinsam orchestrieren, um das Fundament in der medizinisch-klinischen Versorgung und der forschenden Medizin zu verbreitern.

Literatur

[1] FACT SHEET: President_Obama's Precision Medicine Initiative. whitehouse.gov. January 30, 2015. Retrieved April 29, 2016

[2] Collins FS, Varmus H. A New Initiative on Precision Medicine. N Engl ] Med 2015; 372: 793 - 795

[3] Wittgenstein L. Tractus logico-philosophicus. In: Werkausgabe in acht Bänden. Bd. 1 Frankfurt am Main, 1984: 4

[4] Nationale Akademie der Wissenschaften Leopoldina, acatech - Deutsche Akademie der Technikwissenschaften, Union der deutschen Akademien der Wissenschaften (Hrsg). Individualisierte Medizin - Voraussetzungen und Konsequenzen. 2014; 3

[5] Stock G. Möglichkeiten und Grenzen wissenschaftlicher Politikberatung. In: Berlin-Brandenburgische Akademie derWissenschaften, (Hrsg) Gegenworte. Grenzen der Wissenschaft. Berlin: Akademie Verlag; 2012: $9-11$

[6] European Society of Radiology. Medical imaging in personalized medicine: a white paper of the research committee of the European Society of Radiology (ESR). Insights into Imaging 2011; 2: 621 - 630

[7] Bischof Delaloye A, Carrió I, Cuocolo A et al. White paper of the European Association of Nuclear Medicine (EANM) and the European Society of Radiology (ESR) on multimodality imaging. Eur J Nucl Med Mol Imaging 2007; 34: $1147-1151$ 


\section{BILDGEBENDE VERFAHREN}

\section{Medizintechnische Wegbereiter für eine Individualisierte} Diagnostik

Am Anfang einer jeden medizinischen Versorgung, steht die Diagnose. Über die Anamnese im Patient-Arzt-Gespräch hinaus bietet die moderne Medizin heute ein breites Spektrum an diagnostischen Verfahren, welche dank innovativer medizintechnischer Entwicklung eine immer präzisere Darstellung der individuellen, biomedizinischen Eigenschaften eines Patienten und seiner spezifischen Erkrankung ermöglichen. Dabei werden die medizinische Bildgebung und die In-vitroLabordiagnostik genutzt, um erkrankungsrelevante Biomarker zu identifizieren. Diese bilden die Grundlage für die Planung, Durchführung und Überwachung einer individuell am besten geeigneten Therapie. Bildgebung und In-vitro-Diagnostik dienen dabei einerseits als Enabler-Technologien für die molekulargenetisch-basierte Individualisierung, ermöglichen jedoch auch eigenständige Ansätze einer Individualisierten Medizin. Die intelligente Verarbeitung und verantwortungsvolle Nutzung der diagnostischen Daten ist schon heute nicht mehr ohne die Hilfe moderner Informations- und Kommunikationstechnologien möglich.

\section{Bildgebende Verfahren}

Die Bildgebung hat in der diagnostischen Individualisierten Medizin eine herausragende Bedeutung, die sich von der Prävention bis zur Rehabilitation durch alle Bereiche der medizinischen Versorgungkette zieht. Die Signale und Daten der Bildgebung stellen als lokale Biomarker biologische Prozesse im Individuum dar, sowohl im frühen Stadium einer Erkrankung, als auch in der Verlaufs- und Erfolgskontrolle einer Therapie. Dabei werden Diagnostik und Therapie immer häufiger nach dem Prinzip der Theranostik zusammengeführt, beispielsweise in der Radioonkologie ${ }^{1}$. Die Information der Bildgebung bestimmt die Therapie, sowohl bezüglich der Indikation, als auch der Änderung, des Abbruchs oder der Vermeidung.

Vom semantischen Verständnis her bedeutet jede bildgebende Untersuchung am Menschen Individualisierte Medizin, da hier individuelle anatomische, physiologische, zelluläre und molekulare Informationen erfasst werden. Je nach Bedarf ist für den Patienten so eine Basis für eine multimodale und multiparametrische Diagnostik gegeben, die zusammen mit einer molekulargenetischen Diagnostik mittels Omics-Technologie eine präzise Stratifizierung in therapierelevante Untergruppen ermöglicht. Ein Vorteil der Bildgebung besteht in der weitgehend vom Untersucher unabhängigen Darstellung von Biomarkern. Diese kann von Experten beurteilt werden und zu einer wissens- und erfahrungsbasierten Therapieentscheidung führen.

Werden bei der Bildgebung neben den drei räumlichen Dimensionen (3 D) auch Zeitverläufe berücksichtigt (4D) und dabei mehrere, verschiedene Messgrößen als Bildinhalte erfasst, entstehen sehr große Datensätze, die Morphologie,
Histologie, funktionelle Vorgänge und molekularbiologische Prozesse charakterisieren. Diese Datensätze bilden zusammen mit molekulargenetischen Informationen die Grundlage für die Erstellung individualisierter Patientenmodelle. Die Informationsverarbeitung und -nutzung stellt die Lebenswissenschaften wie auch die Informations- und Kommunikationswissenschaften vor große Herausforderungen. Dabei wirkt sich die Vielzahl der heutigen diagnostischen Möglichkeiten erschwerend auf die Auswahl der richtigen Untersuchungsmethode aus, besonders vor dem Hintergrund der individuellen klinischen Fragestellung. An dieser Stelle müssen entsprechende Leitlinien zum Einsatz kommen, welche die Qualität des Untersuchungsergebnisses, die Belastung des Patienten und auch den finanziellen Aufwand gegeneinander abwägen.

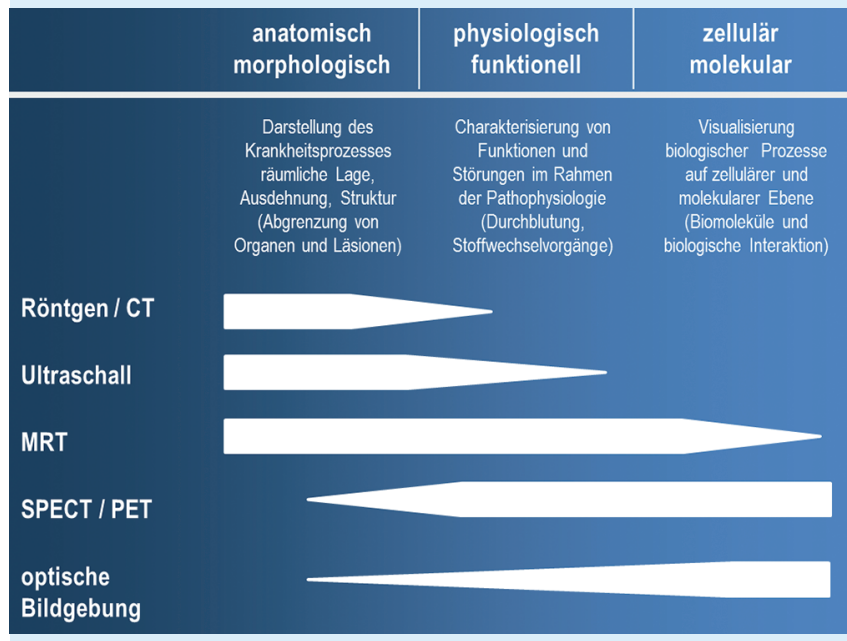

Bildgebende Verfahren in den verschiedenen Dimensionen der Individualisierung

Im Folgenden soll der Schwerpunkt auf Innovationen in der Bildgebung im Kontext der verschiedenen biomedizinischen Techniken gelegt und vor dem Hintergrund der Individualisierten Medizin diskutiert werden.

Ultraschall

Die Ultraschalldiagnostik ist die in der Medizin am häufigsten angewandte Bildgebungsmodalität ${ }^{2}$. Mit der klassischen Echosonografie und den Zusatzmodalitäten Farbdoppler und Elastografie liefert sie als nichtbelastende und kostengünstige Echtzeitmodalität morphologische, funktionelle und Gewebecharakterisierende Informationen. Die Palette derzeit verfügbarer Ultraschallsysteme reicht von universellen Standardgeräten über hochfrequente Spezialsysteme bis hin zu handlichen, mobilen Kleingeräten.

Als Wegbereiter der Individualisierten Medizin spielt der Ultraschall eine wichtige Rolle bei der Bestimmung spezieller und individueller Biomarker, die für Prävention, Prognose und Therapiemaßnahmen nützlich sind. Beispiele sind morphologische und funktionelle Merkmale der Arterien, die Stratifizie- 
rungskonzepte im Zusammenhang mit der Bekämpfung der Arteriosklerose erlauben ${ }^{3}$. Die Bestimmung differenzierter gewebetypischer Merkmale im Rahmen des quantitativen Ultraschalls hat zwar noch nicht Einzug in die allgemeine klinische Routine gehalten, ihr erfolgreicher Einsatz bei der Kontrolle von Verfahren einer Tumortherapie wurde aber nachgewiesen ${ }^{4}$. Für die multimodale Bildgebung sind dedizierte, automatisierte Ultraschallsysteme für bestimmte Organe (z. B. für die weibliche Brust) interessant. Die häufig diskutierte Fusion solcher Systeme mit der Röntgen-Mammografie erlaubt die Stratifizierung bei Brust-Screening-Prozeduren, womit die Strahlenbelastung reduziert und unnötige Biopsien vermieden werden können.

Der Schwerpunkt der Erforschung und Entwicklung medizintechnischer Verfahren und Systeme mit der Perspektive einer Individualisierten Medizin liegt im Bereich der Therapie. Konzepte der Theranostik sind dabei wegen der engen Verknüpfung mit begleitenden diagnostischen Verfahren besonders interessant ${ }^{5}$. Bei der molekularen Bildgebung, die als Grundlage für den Entwurf individualisierter Präventions- und Therapieverfahren zu verstehen ist, kommt auch dem Ultraschall eine erhebliche Bedeutung zu. Ein großes Potenzial ist beim therapeutischen Ultraschall mit seinen thermischen und mechanischen Wirkungen auf Gewebe und Gefäße zu sehen. So lassen sich spezielle Biomarker nach Einwirkung von Ultraschall auf das Gewebe, zum Teil auch in Wechselwirkung mit Mikrobläschen, besser lokalisieren und bestimmen ${ }^{6}$. Die Wechselwirkung von fokussiertem Ultraschall mit Wirkstoff-transportierenden Mikrobläschen oder Nanopartikeln erlaubt zudem die zeitlich und räumlich gesteuerte Freisetzung pharmazeutischer Wirkstoffe (drug delivery), z. B. für eine lokalisierte und in hohem Maße individualisierte Chemotherapie. Risiken bezüglich des Abbaus der Wirkstoffträger im Körper und ihrer Interaktion mit dem Immunsystem sind allerdings noch nicht erschöpfend erforscht ${ }^{7}$. Computertomografie und Magnetresonanztomografie

Die Schnittbildverfahren Computertomografie (CT) und Magnetresonanztomografie (MRT) generieren dreidimensionale Datensätze mit anatomischen und physiologischen Biosignalen und -markern, die eine hochaufgelöste Darstellung des menschlichen Körpers sowie seiner Organsysteme ermöglichen und damit zu einer bedeutenden Grundlage für eine Individualisierte Diagnostik und Therapie werden.

Bei der CT ermöglichen dezidierte Detektorsysteme die spektrale Auswertung der gemessenen Strahlung und damit eine spezifische Gewebsanalytik. Im therapeutischen Kontext wird die CT zur perkutanen Biopsie, Drainage, zur Durchführung thermoablativer Verfahren und zur Planung der Strahlentherapie genutzt ${ }^{8}$. Innovative Rekonstruktionsalgorithmen, spezifi-

3 Vgl. FDA 2013.

4 Vgl. Sadeghi-Naini et al. 2013.

5 Vgl. Kiessling et al. 2014.

6 Vgl. Forbrich et al. 2013.

7 Vgl. jo et al. 2016.

8 Vgl. Prokop et al. 2011. sche Niedrigdosisprotokolle oder gewichtsadaptierte Dosisveränderungen ermöglichen eine Reduktion der Strahlendosen. Dieser strahlenbiologisch begründeten Herausforderung stellen sich Anwender und Hersteller von Geräten zunehmend, insbesondere im Bereich der Screening-Verfahren. Innovationen zielen auf die Etablierung dedizierter Organscanner zum Beispiel von Brustscannern. Mobile CT-Einheiten sind intraoperativ wie auch außerhalb von Krankenhäusern mit Methoden und Strukturen der Telemedizin verfügbar (stroke mobile). Sie müssen zunehmend und sektorübergreifend einen Platz in der flächendeckenden (Erst-) versorgung von Patienten einnehmen und können so zu einer Gesundheitsversorgung betragen, welche die individuellen Lebensumstände der Patienten besser berücksichtigen kann. Aktuell werden in schneller Folge neu- oder weiterentwickelte Systeme auf den Markt gebracht, bevor ihre Vorgänger flächendeckend zum Einsatz kommen konnten. Aus diesem Grund stehen häufig keine prospektiven, klinischen Studienergebnisse zur Verfügung, was die Standardisierung, insbesondere jedoch auch die Nutzenbewertung im Sinne einer Verbesserung der Gesundheitsversorgung zu einer großen Herausforderung werden lässt.

Die MRT detektiert das Resonanzsignal der Wasserstoffatomkerne (Protonen) in einem externen Magnetfeld nach Hochfrequenzanregung. Dieses nicht ionisierende Verfahren liefert über die aus der CT bekannte anatomische Information hinaus biologische Information. Zum Beispiel erlaubt es die Darstellung der Blutversorgung von Organen oder die spektroskopische Erfassung von Stoffwechselprodukten zur Gewebscharakterisierung. Zusätzlich liefern zeitlich aufgelöste Darstellungen der Gefäße individualisierte Informationen zu Krankheitsprozess und Therapiekontrolle.

Jede MRT-Untersuchung erlaubt den Untersuchenden zahlreiche Freiheitsgrade der messtechnischen Gestaltung und individuelle Protokolle. Zunehmend werden quantitative Untersuchungsmethoden des Signalmusters eingesetzt, die eine intra- und interindividuelle parametrische Gewebecharakterisierung erlauben ${ }^{9}$.

Aktuelle Entwicklungen der MRT richten sich auf Schnellbildtechniken, Mehrkanalspulen, quantitative Bildgebung und Kontrastmittel zur Gewinnung individueller metabolischer Informationen, sowie die Evaluation der interventionellen MRT oder die Entwicklung von Höchstfeldsystemen. Letztere werden jedoch nur experimentell in der Forschung, nicht jedoch in der klinischen Versorgung zum Einsatz kommen, da hohe Magnetfeldstärken über acht Tesla nicht unerhebliche Nebenwirkungen für die Patienten hervorgerufen haben.

1.1.1 Einzel-Photonen-Emissions-Computertomografie und Positronen-Emissionstomografie

Die Positronen-Emissionstomografie (PET) und die Einzelphotonen-Emissions-Computertomografie (SPECT) bieten die Möglichkeit, durch externe Detektion die Verteilung und den zeitlichen Verlauf eines radioaktiv markierten Biomarkers an einem molekularen Ziel im Körper eines Patienten dreidimensional darzustellen. Die Gabe geringer Mengen eines träger- 
freien Biomarkers im pico- oder nanomolekularen Bereich vermeidet die biologische Interaktion des Biomarkers mit dem Ziel. Daraus lassen sich Parameter bestimmen, die zu einer individuellen Charakterisierung des Gewebes auf molekularer Ebene führen ${ }^{10}$. Möglichkeiten der Stratifizierung ergeben sich aus der engen Verzahnung zwischen definierter Fragestellung, Markierung identifizierter und interessierender Moleküle, der Detektionstechnik, sowie Bilderstellung und -analyse ${ }^{11}$. SPECT- und PET-Signale werden genutzt, um im Patienten ein frühes Ansprechen auf eine Therapie zu detektieren oder sogar vorauszusagen ${ }^{12}$. Eine Weiterentwicklung besteht in theranostischen Einsatzmöglichkeiten mit Biomarkern, die gleichzeitig eine diagnostische und eine therapeutische Qualität aufweisen. Mit markiertem, diagnostisch und therapeutisch wirksamem PSMA steht beispielsweise eine innovative Versorgung bei Prostatakarzinomen zur Verfügung ${ }^{13}$. Hybride PET/CT- und SPECT/CT-Systeme werden in der klinischen Routine eingesetzt, um die Diagnostik weiter zu verbessern. Darüber hinaus bietet die große Vielfalt an anatomischen und physiologischen Messungen mittels MRT in Kombination mit molekularen Signalen der PET neue Möglichkeiten der nicht-invasiven Charakterisierung von Geweben im Ganzkörper-Modus (PET-MRT) ${ }^{14}$. Erweiterte Perspektiven finden sich in der Anwendung spektroskopischer Techniken mit der sensitiven Detektion von Gamma-Strahlen und MRT-Signalen ${ }^{15}$.

Herausforderungen bestehen in der bisher mangelnden Möglichkeit einer flächendeckenden, sektorenübergreifenden Anwendung der Schnittbildverfahren in der Versorgungsmedizin unter Berücksichtigung ökonomischer Randbedingungen. $\mathrm{Zu}$ den Herausforderungen zählt auch die zeitliche Dynamik der technischen Entwicklung, die nicht allein über klinische Studien bewertet werden kann.

\subsubsection{Optische Bildgebung}

Durch die Erforschung und Weiterentwicklung der hochauflösenden optischen Mikroskopie bis hin zu subzellulärer Auflösung hat sich für die optische Bildgebung in den letzten zwei Jahrzehnten ein breites Portfolio an Anwendungsmöglichkeiten entwickelt ${ }^{16}$.

Innovative Methoden der optischen Bildgebung ermöglichen eine individualisierte In-vivo-Histologie zur Online-Diagnostik und Therapiekontrolle (Theranostik). Neben der biomedizinischen Forschung werden optische Reflexionsverfahren wie die optische Kohärenztomografie (OCT) und die Konfokalmikroskopie zunehmend im klinischen Bereich eingesetzt. Sie erlauben beispielsweise die individuelle Charakterisierung verschiedener Erkrankungen der Retina und haben darüber hinaus im Rahmen endoskopiegestützter Anwendungen zur Entwicklung minimalinvasiver Diagnostik beigetragen. Auch

10 Vgl. Mankoff 2016.

11 Vgl. Schober/Heindel 2010.

12 Vgl. Engert et al. 2012.

13 Vgl. Haberkorn et al. 2016.

14 Vgl. Bailey et al. 2015.

15 Vgl. Zheng et al. 2016.

16 Vgl. Alves/Kiessling 2014. im Bereich der Neurochirurgie werden intraoperativ mikroskopisch Tumorresektionsränder mit Farbstoffen erfasst ${ }^{17}$.

Die spezifische Gewebecharakterisierung wird durch den Einsatz von Autofluoreszenz und fluoreszierenden Farbstoffen erreicht. Dabei können multidimensionale Aussagen von der subzellulären bis zur Gewebeebene getroffen werden (OCT, Multiphotonenmikroskopie, Fluoreszenztomografie). So können insbesondere in der Onkologie die diagnostische Sicherheit erhöht, das therapeutische Vorgehen präzisiert und somit das Auftreten therapiebegleitender Nebenwirkungen wie Funktionsstörungen minimiert werden.

Im Rahmen der Individualisierten Medizin spielen die optische und die fotoakustische Bildgebung für die intraoperative, die endoskopische Anwendung und die Untersuchung von Pathologien an Oberflächen eine große Rolle.

Neue bildgebende Verfahren für die Klinik

Zu den heute etablierten bildgebenden Verfahren können in Zukunft neue Modalitäten hinzukommen, die eine bessere Diagnostik ermöglichen und erweiterte Optionen für eine Individualisierte Medizin bieten. Besonders intensiv werden zurzeit die im Folgenden beschriebenen Systeme erforscht.

Das Phasenkontraströntgen zeigt in präklinischen Untersuchungen einen deutlich höheren Weichteilkontrast im Vergleich zum konventionellen Röntgen. Die Mammografie mit Phasenkontraströntgen könnte die Früherkennung von Brustkrebs deutlich verbessern ${ }^{18}$.

Die Impedanztomografie kann bereits im frühen Stadium einer Erkrankung pathologische Gewebsveränderungen durch Messungen des elektrischen Widerstands detektieren. Die Methode kann zudem zur individualisierten Optimierung der künstlichen Beatmung dienen ${ }^{19}$.

Beim Magnetic Particle Imaging (MPI) werden magnetische Nanopartikel in den Körper eingebracht und dort mit hoher räumlicher und zeitlicher Auflösung verfolg ${ }^{20}$. In der Klinik wird diese Methode zur Darstellung des Gefäßsystems erprobt. Langfristig wird eine Funktionalisierung der Nanopartikel angestrebt, die eine gewebespezifische molekulare Diagnostik ermöglichen soll.

Bei der Abbildung bioelektrischer Quellen werden elektrophysiologische Vorgänge im Körper mit hoher zeitlicher Auflösung dargestellt (z. B. EKG oder EEG). Analog wird die Messung biomagnetischer Felder erprobt ${ }^{21}$. In der Neuromedizin stehen dabei fokale Epilepsien im Vordergrund, in der Kardiologie werden Reizweiterleitungsstörungen erforscht, wie sie bei Herzrhythmusstörungen beobachtet werden.

Eine schnell voranschreitende klinische Translation, von der Präklinik bis zum individuellen Patienten, erfahren derzeit optische Reflexionsverfahren. Diese gestatten eine individuelle In-vivo-Gewebecharakterisierung in der bildgestützten

17 Vgl. Stummer et al. 2006.

18 Vgl. Gromann et al. 2016.

19 Vgl. Frerichs et al. 2014.

20 Vgl. Buzug 2013.

21 Vgl. Dössel 2016. 
Chirurgie und bei endoskopischen Interventionen (s. Kap. 2.1.4 Optische Bildgebung).

Ein Beispiel sind Anwendungen mit Licht im Bereich des nahen Infrarots (NIR). Das Licht dringt einige Zentimeter tief in den Körper ein und kann dazu genutzt werden, Weichteilgewebe im Körper abzubilden und zu charakterisieren. Auch können aktive Bereiche im Gehirn sichtbar gemacht werden. Des Weiteren kann die Diagnostik der Brust durch die sogenannte NIR-Mammografie bei der Fragestellung der Krebsfrüherkennung zum Einsatz kommen und den diagnostischen Wert erhöhen ${ }^{22}$.

Weitere Forschungsfelder betreffen optische Hybridbildgebungsverfahren. So verspricht beispielsweise die sogenannte fotoakustische Bildgebung eine hohe Eindringtiefe und kann neben Kontrastmitteln auch endogene Kontraste (Blut, Sauerstoffsättigung, Melanin) erfassen. Eine erste klinische Studie zur Wertigkeit beim Lymphknoten-Staging bei Melanomen wurde publiziert ${ }^{23}$.

Um intraoperativ exakt zu beurteilen, ob ein operativ entfernter Bereich dem im PET auffälligen Gewebebereich entspricht, könnte zukünftig die sogenannte Cerenkov-Bildgebung in Kombination mit PET zum Einsatz kommen ${ }^{24}$. Weitere Studien zur Prüfung der klinischen Zuverlässigkeit sind jedoch noch nötig.

Auszug aus dem Positionspapier: acatechPOSITION_IndivMed-durch-MedTech-14_06_2017.doc

Auszug aus: acatech (Hrsg.) Individualisierte Medizin durch Medizintechnik (acatech POSITION PAPER). München: Herbert Utz (im Druck)

\section{Literaturverzeichnis}

Alves F, Kiessling F (Hrsg) (2014) Optical Molecular Imaging. In: Brahme A (Hrsg) Comprehensive Biomedical Physics. Vol. 4, Elsevier, Amsterdam

Attenberger UI, Runge VM, Schneider G et al. Essentials der klinischen MRT. 2011, Georg Thieme Verlag, Stuttgart 2011

Bailey DL, Pichler BJ, Gückel B et al. Combined PET/MRI: Multimodality multiparametric imaging is here. Molecular Imaging and Biology 2015; 17: $595-608$

Buzug T (Hrsg) (2013) Biomedical Engineering/Biomedizinische Technik. In: Special Issue on Magnetic Particle Imaging, 58: 6, Berlin: de Gruyter

Dössel O, Buzug TM. (Hrsg) (2014) Biomedizinische Technik, Band 7 (Medizinische Bildgebung), de Gruyter, Berlin

Dössel O Abbildung bioelektrischer Quellen. In: Dössel O (Hrsg) Bildgebende Verfahren in der Medizin, Wiesbaden: Springer Vieweg 2016, 2. Auflage

Engert A, Haverkamp H, Kobe $\mathrm{C}$ et al. Reduced-intensity chemotherapy and PET-guided radiotherapy in patients with advanced stage Hodgkin's lymphoma (HD15 trial): a randomised, open-label, phase 3 non-inferiority trial. Lancet 2012; 12: 379: 1791 - 1799

Vgl. Poellinger et al. 2011.

Vgl. Stoffels et al. 2015.

Vgl. Yamamoto et al. 2014.
US Food and Drug Administration (FDA): Paving the Way for Personalized Medicine: FDA's Role in a New Era of Medical Product Development. Silver Spring, MD 2013

Forbrich A, Paproski R, Hitt M, Zemp R. Microbubble-enhanced ultrasound liberation of mRNA biomarkers in vitro. Ultrasound in Medicine \& Biology 2013; 39: 1087 - 1093

Frerichs I, Becher T, Weiler N. Methodology of electrical impedance tomography-derived measures of regional lung ventilation. Critical Care 2014; 18: 635

Gromann LB, Bequé D, Scherer K et al. Low-dose, phase-contrast mammography with high signal-to-noise ratio. Biomedical Optics Express 2016; 7: 381 - 391

Haberkorn U, Eder M, Kopka K et al. New strategies in prostate cancer: Prostate-specific membrane antigen (PSMA) ligands for diagnosis and therapy. Clinical Cancer Research 2016; 22: 9 - 15 Jo SD, Ku SH, Won YY, Kim SH, Kwon IC. Targeted nanotheranostics for future personalized medicine: recent progress in cancer therapy. Theranostics 2016; 6: $1362-1377$

Kiessling F, Fokong S, Bzyl J et al. Recent advances in molecular, multimodal and theranostic ultrasound imaging. Advanced Drug Delivery Reviews 2014; 72: 15 - 27

Nationale Akademie der Wissenschaften Leopoldina, Deutsche Akademie der Technikwissenschaften (acatech), Union der deutschen Akademien der Wissenschaften (Hrsg): Individualisierte Medizin - Voraussetzungen und Konsequenzen (Stellungnahme), Berlin: mediabogen - Fiedler-Nöschel GbR 2014 Mankoff DA. Development of Companion Diagnostics. Seminars in Nuclear Medicine 2016; 46: 47 - 56

Pereira GC, Traughber M, Muzic RF. The role of imaging in radiation therapy planning: past, present, and future. BioMed Research International Article ID 2014; 231 090, 9 pages Poellinger A, Burock S, Grosenick D et al. Breast cancer: earlyand late-fluorescence near-infrared imaging with indocyanine green-a preliminary study. Radiology 2011; 258: 409 - 416

Prokop M, Galanski M, Schäfer-Prokop C et al. (Hrsg) (2011) Ganzkörper-Computertomographie. Thieme Verlag, Stuttgart Sadeghi-Naini A, Falou O, Tadayyon $\mathrm{H}$ et al. G) Conventional frequency ultrasonic biomarkers of cancer treatment response in vivo. Translational Oncology 2013; 6: 234- 243 Schober O, Heindel W. (2010) PET-CT Hybrid Imaging. Thieme Verlag, Stuttgart

Stoffels I, Morscher S, Helfrich I et al. Metastatic status of sentinel lymph nodes in melanoma determined noninvasively with multispectral optoacoustic imaging. Science Translational Medicine 2015; 9: 317ra199

Stummer W, Pichlmeier U, Meinel T et al. Fluorescence-guided surgery with 5-aminolevulinic acid for resection of malignant glioma: a randomised controlled multicentre phase III trial. The Lancet Oncology 2006; 7: $392-401$

Yamamoto S, Hamamura F, Watabe T et al. Development of a PET/Cerenkov-light hybrid imaging system. Medical Physics 2014; 41: 092504

Zheng Y, Miller GW, Tobias WA, Cates GD. A method for imaging and spectroscopy using $Y$-rays and magnetic resonance. Nature 2016; 537: $652-655$ 